Pathophysiology of Haemostasis and Thrombosis
Pathophysiol Haemost Thromb 2005;34:188-193

DOI: $\underline{10.1159 / 000092421}$

\title{
Comparison of Textilinin-1 with Aprotinin as Serine Protease Inhibitors and as Antifibrinolytic Agents
}

\author{
Simone Flight ${ }^{a, b}$ Lambro Johnson ${ }^{a}$ Manuela Trabi ${ }^{\text {b, d }}$ Patrick Gaffney ${ }^{c}$ \\ Martin Lavin ${ }^{d}$ John de Jersey ${ }^{b}$ Paul Masci $^{a}$ \\ ${ }^{a}$ School of Medicine, University of Queensland, Princess Alexandra Hospital, Brisbane, ${ }^{b}$ School of Molecular \\ and Microbial Sciences, University of Queensland, Brisbane, Australia; ${ }^{\mathrm{c} S t ~ T h o m a s ' s ~ H o s p i t a l, ~ L o n d o n, ~ U K ; ~}$ \\ ${ }^{\mathrm{d}}$ The Queensland Cancer Fund Research Unit, The Queensland Institute of Medical Research, Royal Brisbane \\ Hospital, Brisbane, Australia
}

\section{Key Words}

Textilinin · Aprotinin • Thrombelastography •

Fibrinolysis · Enzyme inhibition · Plasmin $\cdot$ Kallikrein

\begin{abstract}
Textilinin-1 (08008) was isolated from the venom of the Pseudonaja textilis and has a $47 \%$ sequence identity to the antihaemorrhagic therapeutic agent aprotinin. When equimolar concentrations of enzyme and aprotinin were pre-incubated, plasmin was inhibited $100 \%$, plasma kallikrein $58 \%$, and tissue kallikrein $99 \%$. Under the same conditions, textilinin-1 inhibited plasmin 98\%, plasma kallikrein $16 \%$ and tissue kallikrein $17 \%$. Whole blood clot lysis was inhibited strongly by both aprotinin and textilinin-1, as shown by thrombelastography. At $2 \mu M$ inhibitor lysis initiated by t-PA was greater than $99 \%$ inhibited by aprotinin (LY60 $=0.4 \pm 0.1$ ) whereas textilinin- 1 , inhibited lysis by $91 \%$ (LY60 $=8.9 \pm 0.7)$. The same trend was found with the lysis of euglobulin fractions. From these data textilinin-1 appears to be a more specific plasmin inhibitor than aprotinin but aprotinin inhibits clot lysis to a greater extent.
\end{abstract}

Copyright (c) 2005 S. Karger AG, Basel

\section{Introduction}

Reducing blood loss during surgery is a clinical imperative due to shortages in donor blood supply and the risk of introducing blood borne diseases such as hepatitis, human immunodeficiency virus and variant CreutzfeldtJakob disease from replacement blood.

Textilinin-1 is a $6.7 \mathrm{kDa}$ peptide serine protease inhibitor isolated from the venom of Pseudonaja textilis [1]. It has a similar sequence ( $47 \%$ identity) to the $6.5 \mathrm{kDa}$ peptide aprotinin, isolated from bovine lung, which is widely used as an antibleeding agent. Aprotinin (marketed as Trasylol ${ }^{\circledR}$ ) was identified independently in the 1930s by Kraut et al. [2] and Kunitz and Northrop [3] and was first used for treatment of pancreatitis and severe head injury. Aprotinin is a potent inhibitor of trypsin and the high resolution structure of the aprotinin-trypsin complex has been determined [4, 5]. As well as trypsin, aprotinin inhibits other serine proteases including several involved in haemostasis [6, 7].

The use of aprotinin to reduce blood loss is now common during cardiac surgery [8]. The mechanism by which aprotinin acts are thought to be primarily through its direct inhibition of plasmin [9-11]. The preservation of platelet activity is also thought to contribute to the reduction in blood loss [12-14]. 
More recently aprotinin has been found to be an effective treatment for other conditions such as tendonitis [15], tumours [16] and sepsis [17]. Not all of the observed effects of aprotinin are caused directly by enzyme inhibition and effects on platelet function; some involve more complex interactions, which affect coagulation $[18,19]$ and inflammation [20].

Textililin-1 inhibits plasmin and has been shown to reduce bleeding in a small animal model [1]. However, the specificity of textilinin-1 for other clinically relevant serine proteases and its action as an antifibrinolytic agent in vitro has not been previously reported.

This paper describes experiments measuring the inhibition of plasmin, plasma kallikrein, tissue kallikrein and several other serine proteases as well as examining the effect of textilinin-1 on lysis of clots formed in whole blood and plasma in vitro. These results are used to discuss potential advantages and disadvantages of textililin- 1 over aprotinin as a therapeutic antibleeding agent.

\section{Materials and Methods}

\section{Reagents}

All enzyme activities were measured using an appropriate chromogenic substrate purchased from Chromogenix (Sweden). The substrate codes used for the enzymes were as follows: S-2222 (factor Xa), S-2251 (plasmin), S-2302 (tissue and plasma kallikrein), S-2288 (t-PA), S-2238 (thrombin) and S-2765 (trypsin and tryptase). All other reagents were purchased from Sigma Aldrich (USA) unless otherwise specified.

\section{Enzymes}

Human plasmin was purchased from Laboratoire Choay (France) and reconstituted to $4.6 \mathrm{U} / \mathrm{mg}$ in $50 \mathrm{~m} M$ Tris- $\mathrm{HCl}$ (pH 7.4) containing 50\% glycerol. Plasma kallikrein was purchased from Calbiochem (Germany) at a concentration of $20.4 \mathrm{U} / \mathrm{mg}$. Porcine tissue kallikrein was purchased from Sigma Aldrich at a concentration of $26 \mathrm{U} / \mathrm{mg}$. Trypsin was purchased from Sigma Aldrich and reconstituted to a concentration of $140,000 \mathrm{U} / \mathrm{mg}$. Recombinant tissue plasminogen activator (rt-PA) was obtained from Genentech (USA) as Actilyse ${ }^{\circledR}$. Thrombin, factor Xa and tryptase were purchased from Sigma Aldrich.

\section{Inhibitors}

Aprotinin was purchased as Trasylol ${ }^{\circledR}$ from Bayer Pharmaceuticals (Germany) as a $50 \mathrm{ml}$ sterile aqueous solution consisting of $1.4 \mathrm{mg} / \mathrm{ml}$ protein $(10,000 \mathrm{KIU} / \mathrm{ml})$ in $\mathrm{NaCl} 450 \mathrm{mg}$, $\mathrm{NaOH}$ q.s, $\mathrm{HCl}$ q.s. Recombinant textilinin-1 (textilinin-1), coded Q8008 and produced by BresaGen Ltd. (Australia) has the same amino acid sequence as native textilinin-1 [1]. Q8008 is the product code for the recombinant textilinin-1designated by QRx Pharma Pty, Ltd. Textilinin-1 was stored in $25 \mathrm{mM}$ Tris- $\mathrm{HCl}$ (pH 7.8), $50 \mathrm{mg} / \mathrm{ml}$ mannitol, $0.01 \%$ Tween-20 at $0.8 \mathrm{mg} / \mathrm{ml}$ at $4{ }^{\circ} \mathrm{C}$.

\section{Molecular Modelling of Textilinin-1}

A model of textilinin-1 based on the crystal structure of an aprotinin mutant (K15R, A16L, M52L) in complex with bovine $\beta$-tryp$\sin$ (PDB ID 1 ejm) was created using the modeler module in Insight II (Biosym).

\section{Chromogenic Enzyme Assays}

Each $1 \mathrm{ml}$ assay was formulated in a plastic cuvette and contained $150 \mu M$ of the relevant substrate. The concentration of uninhibited enzyme used for each test was that which resulted in a change of $\sim 0.1$ absorbance units in $10 \mathrm{~min}$. Inhibition experiments were performed by pre-incubating the enzyme and inhibitor at equimolar concentrations for $5 \mathrm{~min}$ at $22^{\circ} \mathrm{C}$. Each test was monitored at $405 \mathrm{~nm}$ for $10 \mathrm{~min}$ and performed in triplicate. The results are expressed as percentage inhibition of the control by the inhibitor-containing tests.

\section{Thromboelastography}

Thromboelastography was performed using a Thrombelasto$\operatorname{graph}^{\circledR}\left(\right.$ TEG $^{\circledR}$, Haemscope Corporation Pty Ltd, USA). The TEG $^{\circledR}$ measures the elastic properties of the whole blood clot during its formation and lysis through impedance. The basic principles of thromboelastography are described by Salooja and Perry [21]. The reaction time $(\mathrm{R})$ is the time from the start to the formation of the first significant levels of a detectable clot. This is the point at which most traditional plasma clotting assays reach their end point. The amplitude (A) is a measurement of the stiffness or strength (shear modulus) of the clot. The maximum amplitude (MA) is the point where the clot is at its strongest. The LY60 value is the percentage lysis $60 \mathrm{~min}$ after MA is reached.

Each clot formation/lysis assay contained $320 \mu \mathrm{l}$ of a citrated whole blood, bovine thrombin (1 U), rt-PA (33 IU), $\mathrm{CaCl}_{2}(20 \mathrm{mM})$ and textilinin- 1 or aprotinin $(2 \mu M)$ to make a total assay volume of $360 \mu \mathrm{l}$. This level of rt-PA (33 IU/assay or $\sim 14 \mathrm{n} M$ ) was chosen as it resulted in the production of plasmin to result in almost full clot lysis over $90 \mathrm{~min}$, allowing the effects of the plasmin inhibitors to be monitored. The concentration of inhibitor chosen $(2 \mu M)$ is similar to the plasma concentration after the dose of aprotinin received in the Hammersmith (low-dose) regime [22]. Each experiment was performed for at least $60 \mathrm{~min}$ after the maximal amplitude was reached to establish the LY60 value. TEG LY60 data are presented in tabular form as averages. Errors are the standard error of the mean and the significance between data sets was determined using the Student $t$ test (paired, 2-tailed distribution). $\mathrm{p}$ values $<0.05$ were considered statistically significant.

\section{Euglobulin Clot Lysis Time}

Euglobulin clot lysis time was performed using a method similar to that described by Lassen [23]. Citrated human plasma was diluted with $\mathrm{H}_{2} \mathrm{O}$ at $4^{\circ} \mathrm{C}$ ( $9 \mathrm{ml}$ of $\mathrm{H}_{2} \mathrm{O}$ to $1 \mathrm{ml}$ of plasma) and adjusted with $1 \%$ acetic acid to $\mathrm{pH} 5.9$ then incubated at $4^{\circ} \mathrm{C}$ for $1 \mathrm{~h}$. The plasma was transferred to test tubes $(10 \mathrm{ml}$ per tube $)$ and centrifuged $(10,000 \mathrm{~g}$ for $20 \mathrm{~min})$. The supernatant was decanted, discarded and the tubes inverted for $15 \mathrm{~min}$. The insides of the tubes were dried with swabs and each pellet was resuspended in $80 \mathrm{~m} M$ barbitone buffer $\mathrm{pH}$ 8.6. Inhibitors $(0.4 \mathrm{n} M)$ were added as appropriate. Bovine thrombin $(1 \mathrm{U})$ was added to clot the euglobulin fractions. Tubes were incubated at $37^{\circ} \mathrm{C}$ and lysis monitored. All clots were weighed when full lysis of the control was first evident. 


\section{Results}

\section{Enzyme Inhibition by Textilinin-1 and Aprotinin}

Table 1 illustrates the percentage inhibition of trypsin and three enzymes important in haemostasis and the inflammatory response by equimolar concentrations of textilinin-1 and aprotinin. Both compounds were potent inhibitors of trypsin and plasmin at equimolar concentrations of enzyme and inhibitor. Aprotinin was also a strong inhibitor of tissue and plasma kallikrein whereas textilinin-1 was a much weaker inhibitor of these two enzymes. At equimolar concentrations the activity of t-PA, thrombin, fXa and tryptase was not significantly affected by textilinin-1 or aprotinin.

Table 1. Enzyme inhibition by textilinin-1 and aprotinin at equimolar concentrations

\begin{tabular}{lccc}
\hline Enzyme & $\begin{array}{l}\text { Concentration of } \\
\text { enzyme and } \\
\text { inhibitor }(\mathrm{n} M)\end{array}$ & \multicolumn{2}{c}{\begin{tabular}{l} 
Percentage inhibition \\
\cline { 3 - 4 }
\end{tabular}} \\
\cline { 3 - 4 } Trypsin & 18 & $96 \pm 6$ & $100 \pm 0$ \\
Plasmin & 130 & $98 \pm 3$ & $100 \pm 1$ \\
Plasma kallikrein & 45 & $16 \pm 1$ & $58 \pm 2$ \\
Tissue kallikrein & 49 & $17 \pm 2$ & $99 \pm 1$ \\
\hline
\end{tabular}

${ }^{a}$ The rates of substrate hydrolysis by four enzymes pre-incubated with equimolar concentrations of textilinin-1 or aprotinin are presented as percentage inhibition of the uninhibited rate. Each value is the average of the mean of triplicate measurements \pm standard deviation.

No inhibition of thrombin $(270 \mathrm{n} M)$, fXa $(25 \mathrm{n} M)$, t-PA (73 $\mathrm{n} M)$ or tryptase $(0.33 \mathrm{n} M)$ by either inhibitor was detected under the conditions used for this experiment.

\section{Molecular Modelling of Textilinin-1}

Textilinin-1 and aprotinin are both Kunitz-type serine protease inhibitors of 59 and 58 amino acid residues respectively, each with three disulfide bonds in equivalent positions as shown in figure 1.

In an effort to understand the differences in inhibition on a molecular basis, we have modeled textilinin-1 based on the crystal structure of an aprotinin mutant (K15R, A16L, M52L) in complex with bovine $\beta$-trypsin (PDB ID 1ejm) (see fig. 2). The modeling showed that textilinin-1 could adopt a similar structure to aprotinin. The primary interactions between textilinin- 1 and the protease are between the canonical loop of the inhibitor (residues 15-20) and the active site of the enzyme. The positively charged side chain of the P1 residue, Arg in textilinin-1 and Lys in aprotinin, respectively, interacts with the negatively charged Asp189 in the enzyme's specificity pocket. The biggest difference between textilinin-1 and aprotinin within the canonical loop lies at residue P1', a bulky Val in textilinin-1, but an Ala in aprotinin. The structure of trypsin in complex with the aprotinin $(\mathrm{K} 15 \mathrm{R}, \mathrm{A} 16 \mathrm{~L}$, M52L) mutant suggests that these differences may affect the tightness of binding and possibly the protease specificity of the inhibitors. Work is continuing in our laboratory to determine the structure of the textilinin-1-trypsin complex.

\section{Effect of Inhibitors on Fibrinolysis of Clots Formed in} Whole Blood and Plasma

Thromboelastography was performed to analyse the effect of textilinin-1 and aprotinin on the t-PA-stimulated lysis of clots formed in whole blood by addition of thrombin. Figure 3 illustrates examples of the TEG ${ }^{\circledR}$ traces obtained and table 2 lists the LY60 data so generated. With no t-PA or inhibitor added (control, no t-PA) no clot lysis

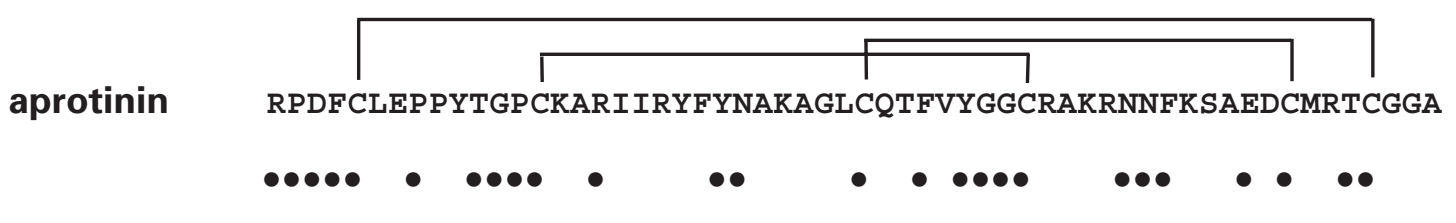

textilinin-1 KDRPDFCELPADTGPCRVRFPSFYYNPDEKKCLEFIYGGCEGNANNFITKEECESTCAA

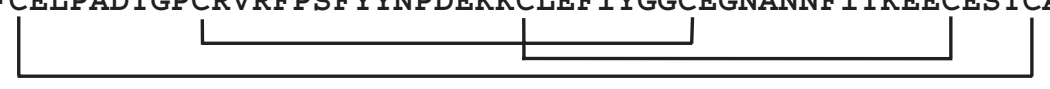

Fig. 1. Structure of aprotinin and textilinin-1. 
could be detected after $180 \mathrm{~min}$ and the LY60 values were $<0.1 \%$. In reactions containing t-PA but no inhibitor, clots were fully lysed after $90 \mathrm{~min}$, with a mean LY60 value of $96.3 \pm 4.4 \%$. $60 \mathrm{~min}$ after the MA, both inhibitors caused a dramatic reduction of fibrinolysis $(\mathrm{LY} 60=0.4 \pm 0.1 \%$ aprotinin and LY60 $=8.9 \pm 0.7 \%$ textilinin-1). The reactions were monitored for $180 \mathrm{~min}$ and at this time the amplitude of the t-PA + textilinin-1

Table 2. Data generated from the thromboelastography illustrated in figure 3

\begin{tabular}{lc}
\hline Experiment & LY60 \\
\hline Control (no t-PA) & $0 \pm 0$ \\
Control (added t-PA) & $96.3 \pm 4.4$ \\
Aprotinin & $0.4 \pm 0.1$ \\
Textilinin-1 & $8.9 \pm 0.7$ \\
\hline
\end{tabular}

Each measurement represents the mean \pm SE of triplicate measurements each using a pool of whole blood from 9 patients. Errors are the standard error of the mean. p-Value for the control (no t-PA) and aprotinin is 0.04 and for aprotinin and textilinin-1 $\mathrm{p}=0.001$.

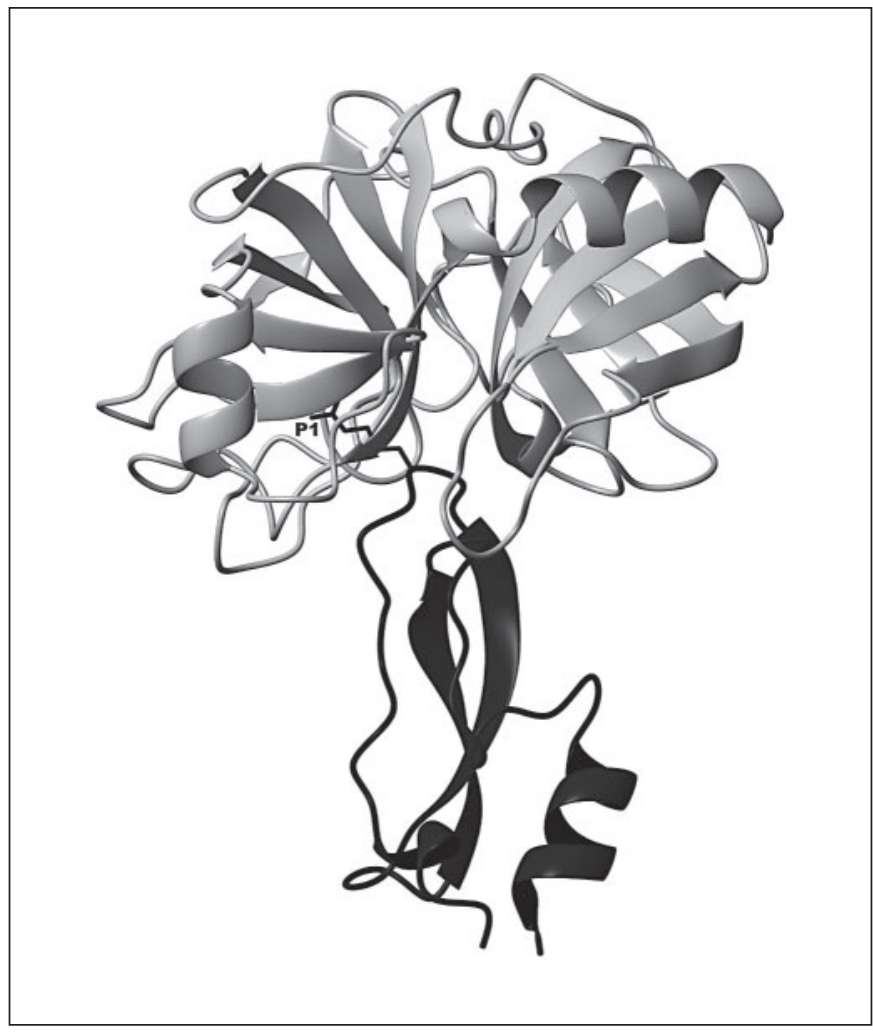

Fig. 2. Ribbon representation of trypsin (light grey) in complex with the model of textilinin-1 (dark grey). The P1 residue is labeled.

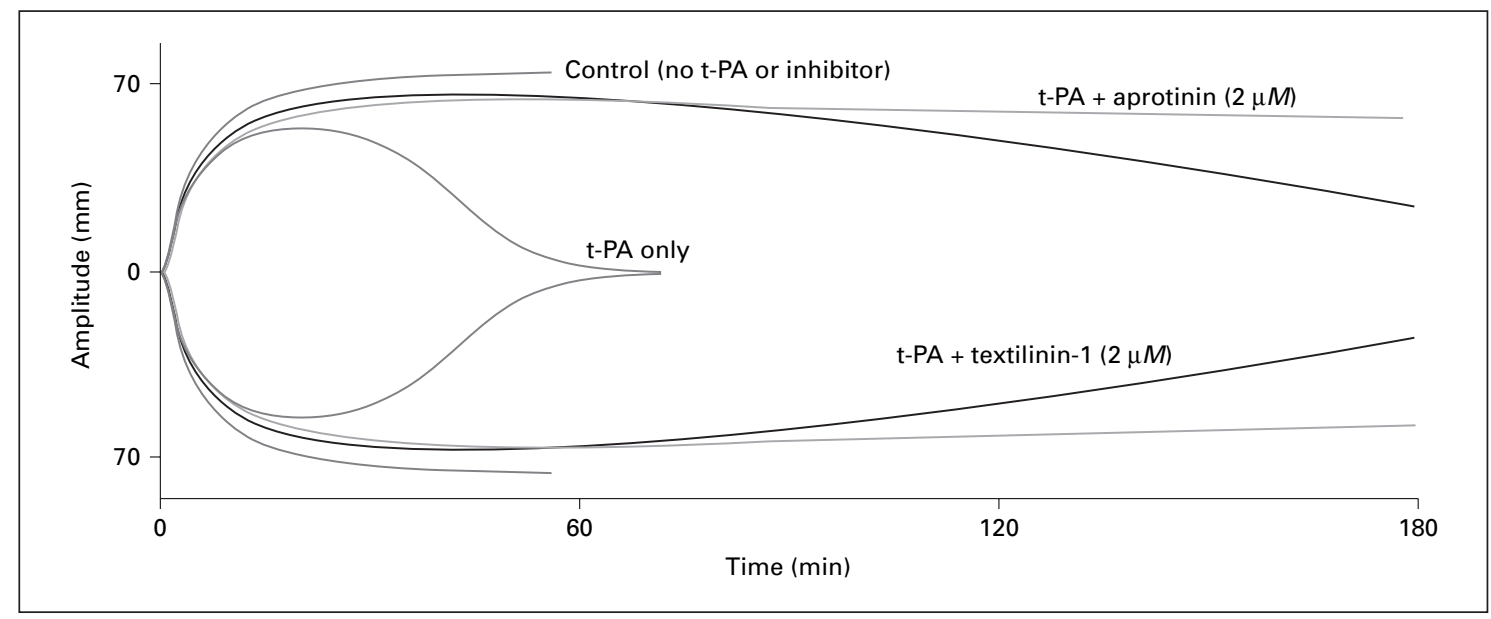

Fig. 3. Thrombelastogram illustrating the overlayed traces from four separate experiments under different conditions. All experiments contained bovine thrombin $(1 \mathrm{U}), \mathrm{CaCl}_{2}(20 \mathrm{mM})$ and citrated whole blood (320 $\left.\mu \mathrm{l}\right)$. 'Control (no t-PA or inhibitor)' contained no additional components, 't-PA only' contained 33 IU of added t-PA, 't-PA + aprotinin $(2 \mu M)$ ' contained 33 IU of t-PA and $2 \mu M$ aprotinin, 't-PA + textilinin-1 (2 $\mu M)^{\prime}$ ' contained 33 IU oft-PA and $2 \mu M$ textilinin-1. Experiments containing textilinin-1 and aprotinin were monitored for $180 \mathrm{~min}$. In all experiments where no t-PA was added there was $<1 \%$ lysis over $180 \mathrm{~min}$ and where no inhibitor was added there was $>98 \%$ lysis over 90 min. 
$(2 \mu M)$ clots was approximately $50 \%$ of the MA. However, the amplitude of the t-PA + aprotinin $(2 \mu M)$ clots was at least $90 \%$ of the MA. Since fibrinolysis in these experiments was induced by t-PA, the antifibrinolytic effects of aprotinin and textilinin-1 were due to inhibition of plasmin.

Euglobulin clot lysis time is a semi-quantitative measurement determined by visualising the time taken for a clot formed in a euglobulin fraction isolated from plasma to lyse (normally 2-4 h). In this experiment the effects of textilinin-1 and aprotinin $(1.5 \mu M)$ on euglobulin clot lysis were determined by weighing the remaining clots in inhibitor-containing experiments when full lysis was first apparent in the control clots. Before incubation (at time zero) the clots weighed $0.88 \pm 0.045 \mathrm{~g}$. When full clot lysis was first apparent in the controls, the aprotinin and textilinin-1-containing clots weighed $0.75 \pm 0.035 \mathrm{~g}$ and $0.37 \pm 0.035$ g respectively.

\section{Discussion}

Thromboelastography and euglobulin clot lysis were techniques to examine the comparative effects of textilinin-1 and aprotinin on the persistence of clots in vitro. The main differences between these two techniques are the source of t-PA (added in the thromboelastography and endogenous in the euglobulin clot lysis) and the presence of the cellular components of whole blood (absent from the euglobulin fraction derived from plasma). In both experiments the clots containing aprotinin were significantly more persistent than those containing textilinin-1. The less persistent textilinin-1-containing clots seen in these experiments indicate fibrinolysis had been inhibited to a lesser extent. This feature may reduce the risk of thrombosis associated with strong antifibrinolytics (such as aprotinin) making it a safer blood loss reduction therapeutic agent. Alternatively, the weaker inhibition of fibrinolysis could be perceived as a disadvantage in vivo possibly resulting in rapid clot lysis and loss of more blood at a comparable dosing range to aprotinin. However, because of its greater specificity for plasmin it may be possible to use higher doses of textilinin-1 without the risk of side effects associated with non-specific enzyme inhibition in a surgical setting. Earlier experiments have shown textilinin-1 to be as effective as aprotinin, at similar concentrations, at reducing blood loss from an arterial bleed in a small animal model [1] suggesting the less prolonged inhibition of fibrinolysis by textilinin-1 does not compromise its capacity to save blood.
There is evidence suggesting specific plasmin inhibitors such as aminocaproic acid and transamic acid may be less effect in reducing blood loss [24, 25] compared to the non-specific inhibitor aprotinin [26]. Recent data strongly suggests that kallikrein inhibition is an important target in reducing blood loss [27]. However, there is also a study showing that increasing the kallikrein inhibitory activity of an aprotinin-like inhibitor does not increase its efficacy in vivo [28]. Consequently the relative roles plasmin, kallikrein and other factors play in surgical bleeding are unknown.

Plasmin itself has broad substrate specificity and is intrinsic to many and varied functions in the body. Most plasmin-mediated effects such as cell invasion, chemotaxis, tissue remodelling, ovulation, migration of tumour and immune cells and wound healing are due to the proteolytic cleavage of extracellular proteins [29-33]. As plasmin is involved in these processes the therapeutic applications for plasmin inhibitors include treatment of the chronic inflammatory diseases such as joint diseases [34, 35], in particular rheumatoid arthritis [36, 37], along with arteriosclerosis [38] and restriction of cell proliferation during cancer [39].

Future research in our laboratory will include more detailed evaluation of textilinin-1 as an inhibitor of serine proteases whose inhibition may have therapeutic significance. Further in vitro studies will include examination of the effects of textilinin-1 in blood coagulation and fibrinolysis and studies using animal models.

\section{Acknowledgement} Ltd.

This research was supported and funded by QRx Pharma Pty.
Flight/Johnson/Trabi/Gaffney/Lavin/ de Jersey/Masci 


\section{References}

1 Masci PP, Whitaker AN, Sparrow LG, et al: Textilinins from Pseudonaja textilis textilis. Characterization of two plasmin inhibitors that reduce bleeding in an animal model. Blood Coagul Fibrinolysis 2000;11:385-393.

$\checkmark 2$ Kraut H, Frey E, Werle E: Uber die Inaktivierung des Kallikreins. Hoppe Seylers Z Physiol Chem 1930;192:1-21.

-3 Kunitz M, Northrop J: Isolation from beef pancreas of crystalline trypsinogen, trypsin, a trypsin inhibitor and intibular trypsin compound. J Gen Physiol 1936;19:991-1007.

-4 Grzesiak A, Helland R, Smalas AO, Krowarsch D, Dadlez M, Otlewski J: Substitutions at the $\mathrm{P}(1)$ position in BPTI strongly affect the association energy with serine proteinases. J Mol Biol 2000;301:205-217.

-5 Cierpicki T, Otlewski J: NMR structures of two variants of bovine pancreatic trypsin inhibitor (BPTI) reveal unexpected influence of mutations on protein structure and stability. $\mathrm{J} \mathrm{Mol}$ Biol 2002;321:647-658.

6 Hoffmann H, Siebeck M, Thetter O, Jochum M, Fritz H: Aprotinin concentrations effective for the inhibition of tissue kallikrein and plasma kallikrein in vitro and in vivo. Adv Exp Med Biol 1989;247B:35-42.

7 Fritz H, Wunderer G: Biochemistry and applications of aprotinin, the kallikrein inhibitor from bovine organs. Arzneimittelforschung 1983;33:479-494.

8 Davis R, Whittington R: Aprotinin. A review of its pharmacology and therapeutic efficacy in reducing blood loss associated with cardiac surgery. Drugs 1995;49:954-983.

-9 Segal H: Aprotinin: pharmacological reduction of perioperative bleeding. Lancet 2000;355: 1289-1290.

10 Longstaff C: Studies on the mechanisms of action of aprotinin and tranexamic acid as plasmin inhibitors and antifibrinolytic agents. Blood Coagul Fibrinolysis 1994;5:537-542.

11 Ray MJ: Aprotinin reduces blood loss after cardiopulmonary bypass by direct inhibition of plasmin. Thromb Haemost 1997;78:10211026.

$\checkmark 12$ Dietrich W: Reducing thrombin formation during cardiopulmonary bypass: is there a benefit of the additional anticoagulant action of aprotinin? J Cardiovasc Pharmacol 1996;27: 50-57.

13 Khan TA, Voisine P, Sandmeyer J, Feng J, Sellke FW: Aprotinin inhibits protease-dependent platelet aggregation and thrombosis. Ann Thorac Surg 2005;79:1545-1550.
14 van Oeveren W, Roozendaal KJ: Aprotinin protects platelets against the initial effect of cardiopulmonary bypass. J Thorac Cardiovasc Surg 1990;99:788-797.

15 Aubin F, Javaudin L, Rochcongar P: Case report of aprotinin in Achilles tendinopathies with athletes. J Pharm Clin 1997;16:270273.

16 Zacharski LR, Hommann M, Kaufmann R: Rationale for clinical trials of coagulation: reactive drugs in hepatocellular carcinoma. Expert Rev Cardiovasc Ther 2004;2:777-784.

17 Cumming A: Acute renal failure and sepsis: therapeutic approaches. Nephrol Dial Transplant 1994;9:159-163.

18 Rabito BW: Aprotinin: a serine protease inhibitor with therapeutic actions: its interaction with ACE inhibitors. Curr Pharm Des 2003;9: 777-787.

19 Chabbat J, Porte P, Tellier M, Steinbuch M: Aprotinin is a competitive inhibitor of the factor VIIa-tissue factor complex. Thromb Res 1993;71:205-215.

20 Mojcik CF, Levy JH: Aprotinin and the systemic inflammatory response after cardiopulmonary bypass. Ann Thorac Surg 2001;71: $745-754$.

21 Salooja N, Perry DJ: Thrombelastography. Blood Coagul Fibrinolysis 2001;12:327-337.

22 Royston D: High-dose aprotinin therapy: a review of the first five years' experience. J Cardiothorac Vasc Anesth 1992;6:76-100.

23 Lassen M: The estimation of fibrinolytic components by means of the lysis time method. Scand J Clin Lab Invest 1958;10:384-389.

24 Casati V, Bellotti F, Gerli C, Franco A, Oppizzi M, Cossolini M, Calori G, Benussi S, Alfieri $\mathrm{O}$, Torri $\mathrm{G}$ : Tranexamic acid administration after cardiac surgery: a prospective, randomized, double-blind, placebo-controlled study. Anesthesiology 2001;94:8-14.

25 Troianos CA, Sypula RW, Lucas DM, D’Amico F, Mathie TB, Desai M, Pasqual RT, Pellegrini RV, Newfeld ML: The effect of prophylactic epsilon-aminocaproic acid on bleeding, transfusions, platelet function, and fibrinolysis during coronary artery bypass grafting. Anesthesiology 1999;91:430-435.

26 Carless PA, Moxey AJ, Stokes BJ, Henry DA: Are antifibrinolytic drugs equivalent in reducing blood loss and transfusion in cardiac surgery? A meta-analysis of randomised head-tohead trials. BMC Cardiovasc Disord 2005;5: 19.
27 Tanakaa KA, Szlama F, Katoria N, et al: Evaluation of a novel kallikrein inhibitor on hemostatic activation in vitro. Thromb Res 2005; 13 : 333-339.

28 Ohri SK, Parratt R, White T, et al: A genetically engineered human Kunitz protease inhibitor with increased kallikrein inhibition in an ovine model of cardiopulmonary bypass. Perfusion 2001;16:199-206.

29 Zabel BA, Allen SJ, Kulig P, et al: Chemerin activation by serine proteases of the coagulation, fibrinolytic and inflammatory cascades. J Biol Chem 2005.

30 Sturzebecher J, Schweinitz A, Schmalix WA, Wikstrom P: Synthetic urokinase inhibitors as potential anti-invasive drugs. IDrugs 2001;4: 677-683.

- 31 Montuori N, Carriero MV, Salzano S, Rossi G, Ragno P: The cleavage of the urokinase receptor regulates its multiple functions. J Biol Chem 2002;277:46932-46939.

- 32 Jenkins GR, Seiffert D, Parmer RJ, Miles LA: Regulation of plasminogen gene expression by

interleukin-6. Blood 1997;89:2394-2403. $\mathrm{P}$, Juhan-Vague I: Formation, inhibition and clearance of plasmin in vivo. Haemostasis 2000;30:204-218.

34 Del Rosso M, Fibbi G, Matucci Cerinic M: The urokinase-type plasminogen activator system and inflammatory joint diseases. Clin Exp Rheumatol 1999; 17:485-498.

35 Kummer JA, Abbink JJ, de Boer JP, et al: Analysis of intraarticular fibrinolytic pathways in patients with inflammatory and noninflammatory joint diseases. Arthritis Rheum 1992;35: 884-893.

36 Judex MO, Mueller BM: Plasminogen activation/plasmin in rheumatoid arthritis: matrix degradation and more. Am J Pathol 2005; 166 : 645-647.

37 Kawakami M, Kawagoe M, Harigai M, et al: Elevated plasma levels of alpha 2-plasmin inhibitor-plasmin complex in patients with rheumatic diseases. Possible role of fibrinolytic mechanism in vasculitis. Arthritis Rheum 1989;32:1427-1433.

- 38 Lijnen HR: Metalloproteinases in development and progression of vascular disease. Pathophysiol Haemost Thromb 2003; 33:275281.

39 Alfano D, Franco P, Vocca I, et al: The urokinase plasminogen activator and its receptor: role in cell growth and apoptosis. Thromb Haemost 2005;93:205-211. 